\title{
$\beta$-Catenin and Yes-Associated Protein 1 Cooperate in Hepatoblastoma Pathogenesis
}

Qian Min, ${ }^{*}$ Laura Molina,${ }^{\dagger}$ Jing Li, ${ }^{\ddagger}$ Adeola 0. Adebayo Michael, ${ }^{\dagger}$ Jacquelyn 0. Russell, ${ }^{\dagger}$ Morgan E. Preziosi, ${ }^{\dagger}$ Sucha Singh, Minakshi Poddar, ${ }^{\dagger}$ Madlen Matz-Soja, ${ }^{\S}$ Sarangarajan Ranganathan, ${ }^{\mathbb{I} \|}$ Aaron W. Bell, ${ }^{\dagger \|}$ Rolf Gebhardt, ** Frank Gaunitz, Jinming $\mathrm{Yu}^{,}{ }^{* \dagger \dagger}$ Junyan Tao, ${ }^{\ddagger}$ and Satdarshan P. Monga ${ }^{\ddagger \|}$

From the Department of Oncology, ${ }^{*}$ Renmin Hospital of Wuhan University, Wuhan, China; the Division of Experimental Pathology, ${ }^{\dagger}$ Department of Pathology, University of Pittsburgh School of Medicine, and Pittsburgh Liver Research Center, Shiyan Taihe Hospital, Affiliated Hospital of Hubei University of Medicine, Shiyan, China; the Department of Neurosurgery, ${ }^{\S}$ University Hospital Leipzig, Leipzig, Germany; the Division of Pediatric Pathology, ${ }^{\circledR}$ Department of Pathology, Children's Hospital, Pittburgh, Pennsylvania; the Institute of Biochemistry, ** Faculty of Medicine, University of Leipzig, Leipzig, Germany; and the Department of Radiation Oncology, ${ }^{\dagger \dagger}$ Shandong Cancer Hospital Affiliated to Shandong University, Shandong Academy of Medical Science, Jinan, China

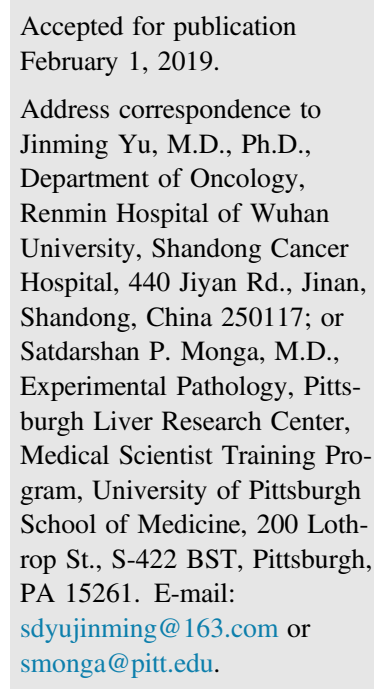

\begin{abstract}
Hepatoblastoma (HB), the most common pediatric primary liver neoplasm, shows nuclear localization of $\beta$-catenin and yes-associated protein 1 (YAP1) in almost $80 \%$ of the cases. Co-expression of constitutively active S127A-YAP1 and $\Delta$ N90 deletion-mutant $\beta$-catenin (YAP1- $\Delta$ N90- $\beta$-catenin) causes HB in mice. Because heterogeneity in downstream signaling is being identified owing to mutational differences even in the $\beta$-catenin gene alone, we investigated if co-expression of point mutants of $\beta$-catenin (S33Y or S45Y) with S127A-YAP1 led to similar tumors as YAP1- $\Delta$ N90- $\beta$-catenin. Co-expression of S33Y/S45Y- $\beta$-catenin and S127A-YAP1 led to activation of Yap and Wnt signaling and development of $\mathrm{HB}$, with $100 \%$ mortality by 13 to 14 weeks. Co-expression with YAP1-S45Y/S33Y- $\beta$-catenin of the dominant-negative T-cell factor 4 or dominant-negative transcriptional enhanced associate domain 2 , the respective surrogate transcription factors, prevented $H B$ development. Although histologically similar, HB in YAP1-S45Y/S33Y- $\beta$-catenin, unlike YAP1- $\Delta$ N90- $\beta$-catenin $H B$, was glutamine synthetase (GS) positive. However, both $\Delta \mathrm{N} 90-\beta$-catenin and point-mutant $\beta$-catenin comparably induced GS-luciferase reporter in vitro. Finally, using a previously reported 16 -gene signature, it was shown that YAP1 $-\Delta N 90-\beta$-catenin HB tumors exhibited genetic similarities with more proliferative, less differentiated, GS-negative HB patient tumors, whereas YAP1-S33Y/S45Y- $\beta$-catenin HB exhibited heterogeneity and clustered with both well-differentiated GS-positive and proliferative GS-negative patient tumors. Thus, we demonstrate that $\beta$-catenin point mutants can also collaborate with YAP1 in HB development, albeit with a distinct molecular profile from the deletion mutant, which may have implications in both biology and therapy. (Am J Pathol 2019, 189: 1091-1104; https://doi.org/10.1016/ j.ajpath.2019.02.002)
\end{abstract}

Although primary liver tumors are rare in children, hepatoblastoma $(\mathrm{HB})$ is the most common primary malignant neoplasm of the liver in children $<5$ years of age and has an increasing incidence of 1.2 to $1.5 /$ million population/year. ${ }^{1}$ In the past several decades, four multi-institutional collaborations, Children's Oncology Group, International Childhood Liver Tumor Strategy Group, German Society of Pediatric Oncology and Hematology, and Japanese Study Group for Pediatric Liver Tumor, have dramatically improved survival from $<20 \%$ to $>70 \%$ for patients without metastatic disease at diagnosis. ${ }^{2}$ Complete tumor resection is the primary radical therapy for HB. In addition, cisplatin-based chemotherapy, used in neoadjuvant and adjuvant treatments, largely decreases the mortality and prolongs overall survival. Moreover, with the improvement

Supported by NIH grants 1R01DK62277 (S.P.M.), 1R01DK100287 (S.P.M.), 1R01CA204586 (S.P.M.), and T32EB0010216 (L.M.); and Endowed Chair for Experimental Pathology (S.P.M.).

Disclosures: None declared. 
in the techniques of liver resection and transplantation, the number of unresectable cases has significantly decreased. ${ }^{3}$ Despite this, patients often present with distant metastatic disease or undifferentiated histologic subtypes, which continue to have a poor prognosis, with 5-year survival at $21 \%$ to $28 \%{ }^{2}$

HB is an embryonic tumor that arises from the bipotent hepatoblasts during liver development and differentiation. ${ }^{4}$ HBs have diverse histologic subtypes, which limits the understanding of their pathogenesis. The major histologic forms of $\mathrm{HB}$ include the following: fetal, crowded fetal, embryonal, macrotrabecular, and small cell (in order from most to least differentiated).

Koch et $\mathrm{al}^{5}$ reported that $48 \%$ of HBs have a high frequency of mutations in the CTNNB1 gene, which encodes for $\beta$-catenin, and the mutations affected exon 3 , which contains key phosphorylation sites that regulate the degradation of $\beta$ catenin, leading to an overaccumulation of cytoplasmic and nuclear $\beta$-catenin. $\beta$-Catenin is a central and critical effector in the Wnt signaling pathway, and it participates in liver development, regeneration, and tumorigenesis through transcriptional regulation of Wnt target genes by binding to T-cell factor (TCF)/lymphoid enhancer-binding factor (LEF) transcription factors. ${ }^{6}$ In previous studies, $25 \%$ of HBs showed point mutations in exon 3 of CTNNB1 and 50\% showed truncation mutations of exon 3 ; both types of mutations lead to $\beta$-catenin activation. ${ }^{7}$ Previous studies have demonstrated differences in the level of $\beta$-catenin activity associated with different mutations that have been observed in adult liver tumors. ${ }^{89}$ Although deletion mutations involving exon 3 and point mutants involving residues within the phosphodegron (eg, S33) exhibit strong $\beta$-catenin activation and are associated with malignant liver tumors, a variety of point mutations at nearby residues, such as $\mathrm{S} 45$, or faraway residues in other domains exhibit weak-to-moderate activation of $\beta$-catenin and are found in both benign and malignant liver tumors. ${ }^{9}$ Some studies have shown that large deletion mutations encompassing exon 3 have been more often associated with fetal $\mathrm{HB}$, whereas small deletions and point mutations are associated with embryonal or other subtypes of $\mathrm{HB} .{ }^{10}$ However, the implications of these differences in HB have not been determined.

At the same time, yes-associated protein 1 (YAP1) activation has been reported in a subset of HBs. ${ }^{11}$ YAP1 is another transcriptional coactivator downstream of the Hippo pathway that partners with the transcriptional enhanced associate domain (TEAD) family of transcription factors to regulate gene expression, promoting cell proliferation and survival; and it has been implicated as a powerful oncogene in numerous cancers. ${ }^{12}$ Because of the complex interplay between Wnt and Hippo signaling pathways, we investigated their relative roles and interdependency in HB. ${ }^{13}$ Previously, we identified nuclear localization of both YAP1 and $\beta$-catenin in approximately $80 \%$ of HB cases from two separate patient cohorts. ${ }^{14}$ On the basis of these results, we developed a mouse model that uses sleeping-beauty (SB) transposase to insert constitutively active mutant-CTNNBI ( $\beta$-catenin) and mutant$Y A P 1$ genes into the genomes of a small subset of hepatocytes, as described previously. ${ }^{14,15}$ Both the plasmids and SB transposase, dissolved in approximately 1 to $1.5 \mathrm{~mL}$ of saline, were injected through tail vein in 5 seconds to overwhelm the cardiac circulation, causing backflow of blood in the inferior vena cava and leading to delivery of the cargo into the lowresistance hepatic vasculature. This well-established method of hydrodynamic tail vein injection (HTVI) induces increased pressure in the highly fenestrated sinusoidal vessels, causing mild hepatocyte damage, and facilitates the uptake of genetic material into a small subset of hepatocytes predominantly in the zone 3 of the hepatic lobule. ${ }^{16,17}$ Previously, using SBHTVI, a deletion-mutant and a constitutively active $\beta$-catenin $(\Delta \mathrm{N} 90-\beta$-catenin) were co-expressed along with a constitutively active YAP1 (YAP1-S127A) in hepatocytes, leading to swift development of HB, with 11-week overall survival. ${ }^{14}$ Herein, it was tested whether two different point mutants of $\beta$-catenin ( $\mathrm{S} 33 \mathrm{Y}-\beta$-catenin or $\mathrm{S} 45 \mathrm{Y}-\beta$-catenin) could similarly interact with constitutively active YAP1 to induce HB. We show that codelivery of either of the point mutants of $\beta$-catenin and YAP1 in mice promotes rapid development of HB. In addition, codelivery of dominantnegative (dn) TCF4 and dnTEAD2 suppressed $\beta$-catenin and YAP1 signaling, respectively, and both prevented the development of HB tumors in the point-mutant $\beta$-catenin and YAP1 model. These results also suggest that point-mutant $\beta$-cateninYAP1 tumors lead to a more differentiated form of $\mathrm{HB}$, as these tumors, unlike the ones driven by $\Delta \mathrm{N} 90-\beta$-catenin, are strongly glutamine synthetase (GS) positive. We identify additional molecular differences between S33Y and S45Y mutants of $\beta$-catenin and $\Delta \mathrm{N} 90-\beta$-catenin in their cooperation with YAP1 in HB pathogenesis.

\section{Materials and Methods}

\section{Animals, Plasmids, and Hydrodynamic Tail Vein Injections}

Wild-type (WT) FVB/N mice were obtained from the Jackson Laboratory (Bar Harbor, ME). SB transposon/transposase plasmids and HTVI have been described previously ${ }^{14}$ and in introduction of this work. ${ }^{18}$ Briefly, $20 \mu \mathrm{g}$ pT3-EF5 $\alpha$-S33Y$\beta$-catenin-Myc-tag and pT3-EF5 $\alpha$-S127A-YAP1-Flag-tag or $20 \mu \mathrm{g}$ pT3-EF5 $\alpha$-S45Y- $\beta$-catenin-Myc-tag and pT3-EF5 $\alpha$ S127A-YAP1-Flag-tag, along with the transposase in a ratio of $25: 1$, were diluted in $2 \mathrm{~mL}$ of normal saline $(0.9 \% \mathrm{NaCl})$; filtered through a $0.22-\mu \mathrm{m}$ filter (Millipore, Burlington, MA); and injected into the lateral tail vein of FVB mice that were approximately 6 weeks old, in 5 to 7 seconds. The injections were also repeated for each group in combination with pT3EF5 $\alpha$-dnTCF4, pT3-EF5 $\alpha$-dnTEAD2, or PT3 plasmid only. Mice were housed, fed, and monitored in accordance with protocols approved by the Institutional Animal Care and Use Committee at the University of Pittsburgh (Pittsburgh, PA). All animals were carefully monitored for signs of morbidity or 
discomfort. Close attention was paid to the abdominal girth. Animals were euthanized at specific time points after injection or at the earliest signs of morbidity.

\section{Immunohistochemistry}

Liver specimens were fixed in $10 \%$ buffer and embedded in paraffin. Hematoxylin and eosin staining on liver sections (4 $\mu \mathrm{m}$ thick) was performed to note the appearance and characteristics of preneoplastic and neoplastic foci at different times after tail vein injection. Immunohistochemistry (IHC) was performed on these sections as well. Formalin-fixed sections (4 $\mu \mathrm{m}$ thick) were deparaffinized in graded xylene and alcohol and rinsed in phosphate-buffered saline. To block endogenous peroxidase activity, the sections were incubated in 3\% hydrogen peroxide (Sigma, St. Louis, MO). For antigen retrieval, slides were microwaved in citrate buffer, followed by blocking with Superblock (ScyTek Laboratories, Logan, UT) for 10 minutes. Sections were incubated overnight at $4^{\circ} \mathrm{C}$ or for 1 hour at room temperature in the following antibodies: cyclin D1 (Rb-9041-P; Thermo Fisher Scientific, Pittsburgh, PA), glutamine synthetase (SC-74430; Santa Cruz Biotechnology, Dallas, TX), Ki-67 (NM-Sp6; Thermo Fisher Scientific), Myctag (Vli01; Maine Medical Center Research Institute, Scarborough, ME), and YAP1 (CS14074; Cell Signaling, Danvers, MA). Sections were then incubated with species-specific secondary horseradish peroxidase-conjugated antibody (Chemicon, Temecula, CA) for 30 minutes at room temperature. Sections stained with antibodies were incubated with streptavidin-biotin, and signal was detected with 3,3'diaminobenzidine (Vector Laboratories, Inc., Burlingame, CA). Then, sections were counterstained with Shandon Hematoxylin (Thermo Fisher Scientific).

\section{Protein Extraction and Western Blot Analysis}

Flash-frozen livers from different treatment group FVB mice were homogenized using radioimmunoprecipitation assay buffer [ $30 \mathrm{mmol} / \mathrm{L}$ tris (pH 7.5), $150 \mathrm{mmol} / \mathrm{L} \mathrm{NaCl}, 1 \%$ nonyl phenoxypolyethoxylethanol (NP-40), $0.5 \% \mathrm{Na}$ deoxycholate, and $0.1 \%$ SDS] containing the protease and phosphatase inhibitor cocktail (Sigma). Tissue homogenate was centrifuged at $20,000 \times g$ for 5 minutes in a cold room. Supernatant was recovered and stored at $-80^{\circ} \mathrm{C}$ for use. Protein concentrations were detected with the Bio-Rad Protein Assay kit (Bio-Rad, Hercules, CA) using bovine serum albumin as a standard. Aliquots of $50 \mu \mathrm{g}$ of proteins were denatured by boiling in Tris-Glycine SDS Sample Buffer (Life Technologies, Carlsbad, CA), resolved by SDS-PAGE, and transferred to polyvinylidene difluoride membranes (Life Technologies) using the Bio-Rad transfer apparatus. Membranes were blocked in 5\% nonfat dry milk or 5\% bovine serum albumin in tris-buffered saline containing $0.1 \%$ Tween-20 for 1 hour. Western blot analysis was performed using the following primary antibodies: cyclin D1 (1:1000, SC-753; Santa Cruz Biotechnology), GS (1:1000, SC-764430; Santa Cruz Biotechnology), survivin (1:1000, CS2808; Cell Signaling), Cyr61 (1:500, SC13100; Santa Cruz Biotechnology), jagged 1 (1:500, SC8303; Santa Cruz Biotechnology), Myc-tag (1:1000, CS 2278; Cell Signaling), and glyceraldehyde3-phosphate dehydrogenase $(1: 1000$, 60004-1-Ig; Proteintech, Rosemont, IL). Membranes were incubated in primary antibodies and diluted in 5\% skim milk or 5\% bovine serum albumin after overnight incubation at $4^{\circ} \mathrm{C}$. Incubation with anti-rabbit or anti-mouse secondary antibody horseradish peroxidase-conjugated IgG (Santa Cruz Biotechnology) was done for 30 minutes at room temperature.

Table 1 List of Primers Used for RT-qPCR in This Study

\begin{tabular}{|c|c|c|}
\hline Gene & Forward primer & Reverse primer \\
\hline $18 S$ rRNA & 5'-GTAACCCGTTGAACCCCATT-3' & 5'-CCATCCAATCGGTAGTAGCG-3' \\
\hline Afp & $5^{\prime}$-GCCTGAACTGACAGAGGAGCA-3' & $5^{\prime}-$ TTTAAACGCCCAAAGCATCAC- $3^{\prime}$ \\
\hline Aldh2 & $5^{\prime}-$ AGGGAGCTGGGCGAGTATG- $3^{\prime}$ & 5'-TGTGTGGCGGTTTTTCTCAGT-3' \\
\hline Apcs & 5'-TGTTTGTCTTCACCAGCСTTCTT-3' & $5^{\prime}$-CGGAAACACAGTGTAAAATTCTGC-3' \\
\hline Apoc4 & $5^{\prime}-\mathrm{AGCCACTGGTGACCAGAACC}-3^{\prime}$ & 5'-AGGAGGTGGTCTCTGGAGCTC-3' \\
\hline Aqp9 & 5'-CCCAGGCTCTTCACTGCTCT-3' & 5'-GGTTCGAGTGATGCATTTGGA-3' \\
\hline Сур2e1 & 5'-TTTCTGCAGGAAAGCGCG-3' & 5'-CTGCCAAAGCCAATTGTAACAG-3' \\
\hline$D \lg 7$ & $5^{\prime}$-ACACCTCTGTCTGCCAGCAA-3' & $5^{\prime}$-GGCACCTGCTTTCAAGACCA-3' \\
\hline Dusp9 & 5'-CAATGTCACCCCCAАCCTTC-3' & $5^{\prime}-\mathrm{ACAGTTCTGCGACAAGGCCT-3^{ \prime }}$ \\
\hline E2f5 & 5'-ACCATGGCTGCTCAAAACCT-3' & $5^{\prime}$-GCCGTAAAAGAGGAAACACATCAG- $3^{\prime}$ \\
\hline Gapdh & 5'-AACTTTGGCATTGTGGAAGG-3' & 5'-ACACATTGGGGGTAGGAACA-3' \\
\hline Rpl10a & $5^{\prime}-$ GGCCTAAACAAGGCTGGCA-3' & $5^{\prime}$-CATCGGTCATCTTCACGTGG- $3^{\prime}$ \\
\hline Rhot2 & $5^{\prime}$-CAACTTGCTACCATGGCCACT-3' & $5^{\prime}-$ ATCGGCTCTTCACTAGGACCC- $3^{\prime}$ \\
\hline
\end{tabular}

RT-qPCR, quantitative RT-PCR. 


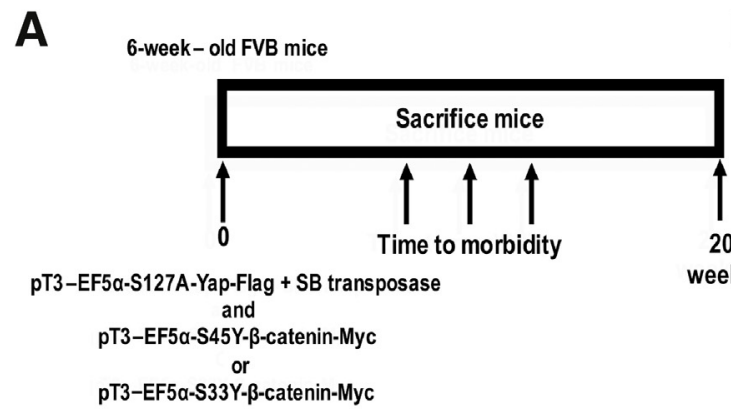

C

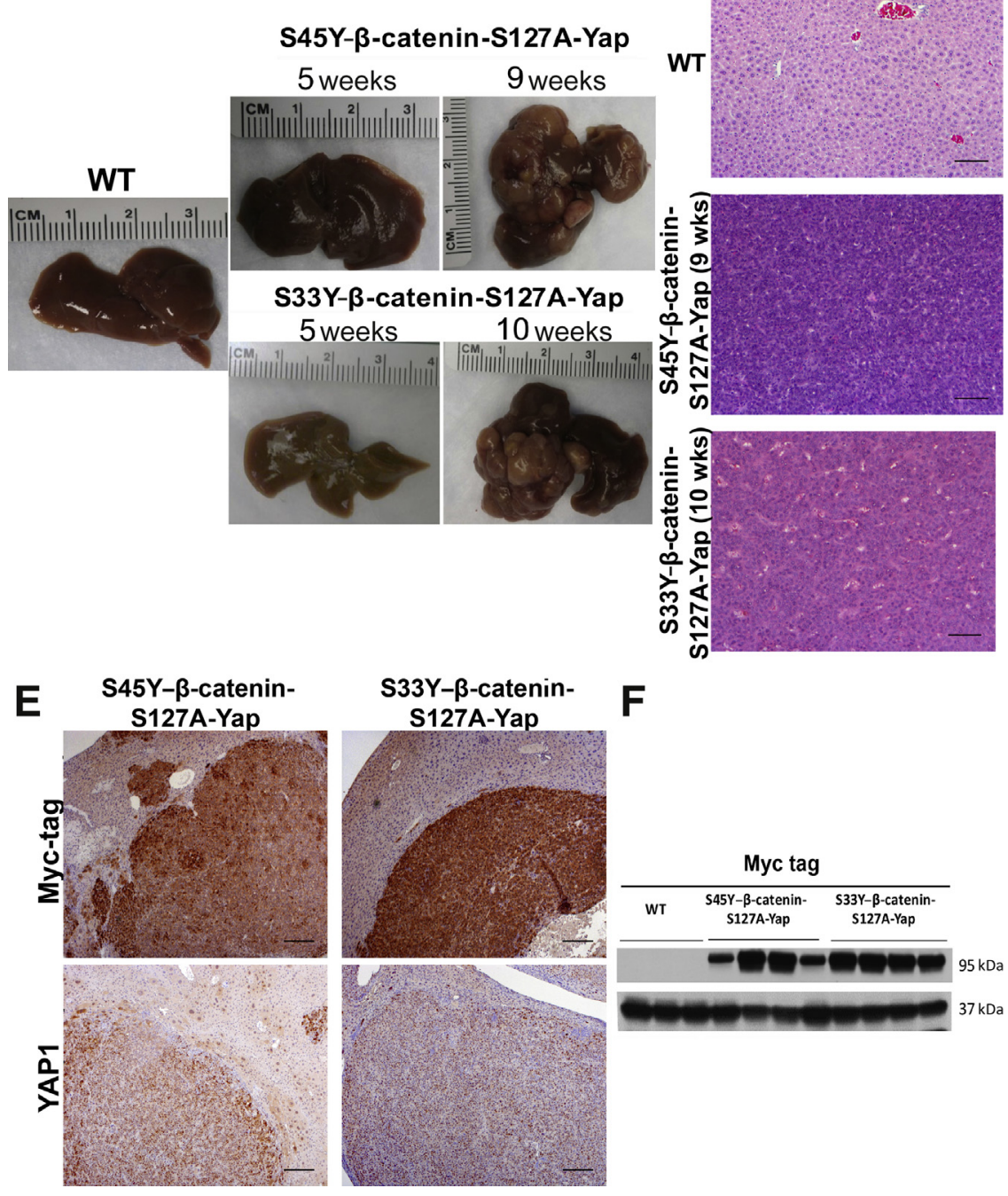

Figure 1 Establishment of a hepatoblastoma (HB) model by co-expression of point-mutant $\beta$ catenin and YAP1. A: Schematic representation of establishment of an HB model, experimental treatments, and timeline. B: Liver weight (LW)/body weight (BW) ratio shows approximately $5 \%$ of BW to be contributed by LW in 10- to 12-week-old wildtype (WT) mice, whereas age-matched S33Y- $\beta$-catenin-S127A-YAP1 at 10 to 12 weeks after sleepingbeauty hydrodynamic tail vein injection (SB-HTVI) and S45Y- $\beta$-catenin-S127A-YAP1 at 8 to 10 weeks after SB-HTVI show, on average, approximately $17 \%$ or $18 \%$ of $\mathrm{BW}$ to be contributed by LW, indicating significant tumorigenesis. C: Gross liver images from S45Y- $\beta$-catenin-S127A-YAP1 and S33Y- $\beta$-cateninS127A-YAP1 mice at 5 and 9 to 10 weeks after SBHTVI, showing progressive macroscopic disease. D: Hematoxylin and eosin (H\&E)-stained sections from WT mouse as well as $S 45 Y-\beta$-catenin-S127A-YAP1 and S33Y- $\beta$-catenin-S127A-YAP1 mice at 9 to 10 weeks after SB-HTVI, showing HB with more differentiated fetal or crowded fetal histology in both tumor groups. E: Immunohistochemistry shows strong nuclear staining of Myc-tag (representing the presence of exogenous point-mutant $\beta$-catenin) and YAP1 in livers from S45Y- $\beta$-catenin-S127A-YAP1 and S33Y- $\beta$-catenin-S127A-YAP1 mice at 9 to 10 weeks after SB-HTVI. F: Western blot analysis for Myctag in S45Y- $\beta$-catenin-S127A-YAP1 and S33Y- $\beta$ catenin-S127A-YAP1 mice at 9 to 10 weeks after SBHTVI. ${ }^{\star} P<0.05$. Scale bars: $100 \mu \mathrm{m}$ (D); $200 \mu \mathrm{m}$ (E). Original magnification: $\times 100(\mathbf{D}) ; \times 50(\mathbf{E})$.
Immunoreactive bands were detected by Super-Signal West Pico Chemiluminescent Substrate (ThermoFisher Scientific, Rockford, IL) and revealed by autoradiography.

\section{Transfection of Reporter Plasmids into Primary Hepatocytes}

The reporter gene $H E V$, originally constructed by Fahrner et $\mathrm{al}^{18}{ }^{18}$ encompasses the glul $5^{\prime}$ enhancer located between
-2156 and -2146 upstream from the glul transcriptional start point in the rat glul gene controlling a Photinus pyralis luciferase gene. This reporter was used to construct the deletion constructs HEV-del TCF, HEV-del STAT5, and HEV-del specificity protein 1 (SP1), containing deletions of the TCF7L2, STAT5, and SP1 binding sites, respectively, as reported previously. ${ }^{19}$ The reporter gene with the $5^{\prime}$ region up to -3780 and down to 105 was constructed by cloning an amplification product from genomic rat DNA, encompassing a 


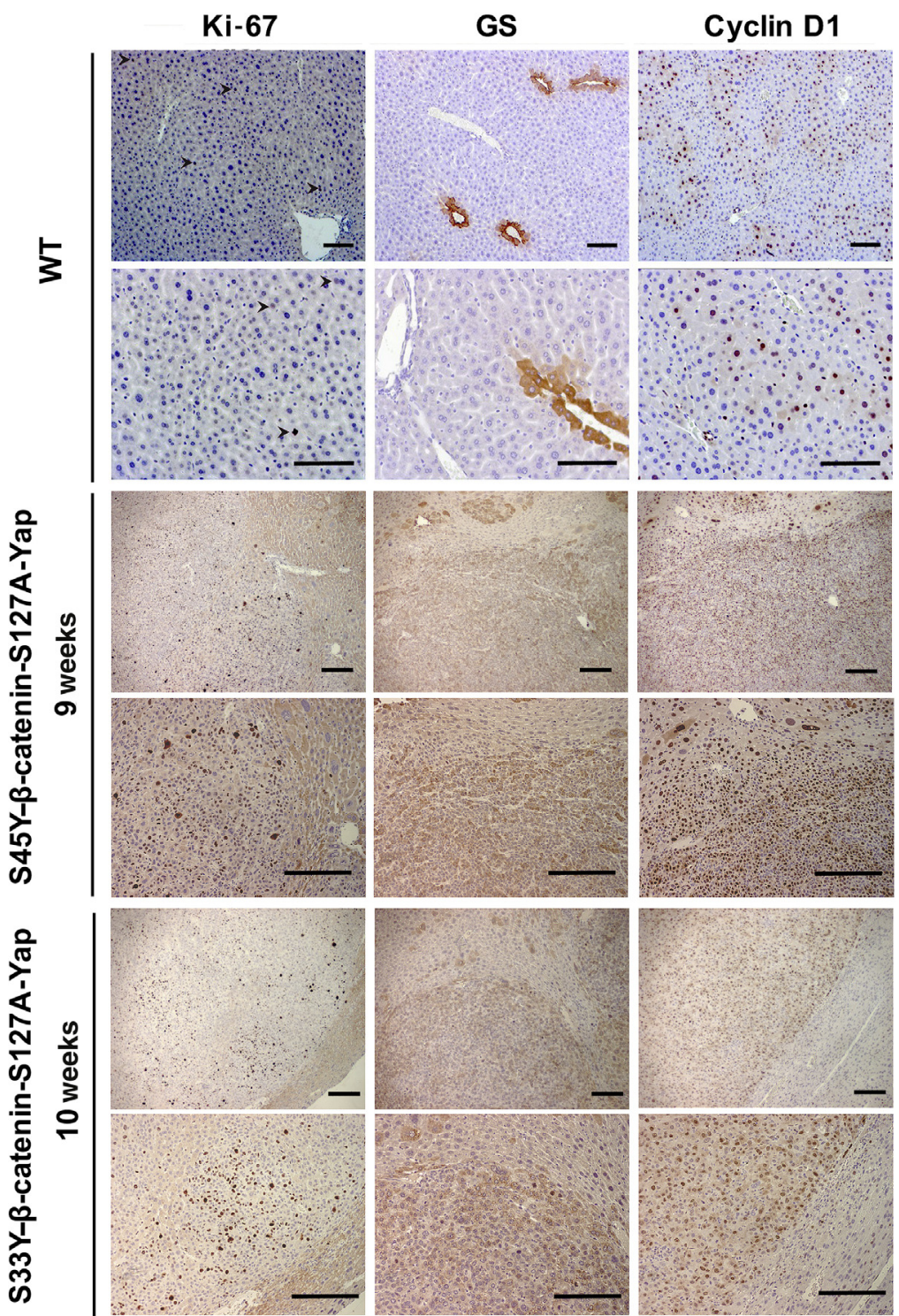

Figure 2 Immunohistochemical characterization of hepatoblastoma (HB) in S45Y- $\beta$-cateninS127A-YAP1 and S33Y- $\beta$-catenin-S127A-YAP1 mice. Representative immunohistochemical analysis of liver sections from wild-type (WT) mice as well as S45Y- $\beta$-catenin-S127A-YAP1 and S33Y- $\beta$-cateninS127A-YAP1 mice at 9 to 10 weeks after hydrodynamic tail vein injection, showing $\mathrm{HB}$ to be positive for proliferation marker $\mathrm{Ki}-67$ and $\beta$-catenin targets glutamine synthetase (GS) and cyclin D1. Arrowheads indicate the few Ki-67-positive cells in the WT liver. Scale bars $=100 \mu \mathrm{m}$. region between the HindIII sites in the rat glul promoter at -3780 and at -2516 , after digestion with HindIII, into a reporter gene that already included an amplification product encompassing the promoter of the rat glul gene between the HindIII site at -2516 and the NaeI site at 105 , cloned into the vector $\mathrm{pXP} 2{ }^{20}$

Hepatocytes isolated from two male C57Bl $/ 6 \mathrm{~N}$ mice, at the age of 12 weeks, were cultivated at 0.01 million. cells per well in 96-well plates in $100 \mu \mathrm{L}$ medium (William's Medium E enriched with $10 \%$ fetal calf serum, $2 \mathrm{mmol} / \mathrm{L}$ L-glutamine, $100 \mathrm{nmol} / \mathrm{L}$ dexamethasone, and penicillin/streptomycin). After 3 hours, medium was changed and transfection was performed using the jetPEI transfection reagent (VWR, Darmstadt, Germany), according to the manufacturer's instruction. For each single plasmid, $100 \mu \mathrm{g}$ DNA per well was used. For control, only the DNA of the vector was transfected into the hepatocytes (mock transfection).
RNA Extraction, Quantitative RT-PCR, and Unsupervised Hierarchical Clustering of Mouse and Human Tumors

Only tumor samples with significant tumor burden (at least $10 \%$ liver weight/body weight ratio) were used for analysis. RNA was extracted from HB tumor samples with TRIzol (Ambion, Carlsbad, CA). A DNAse kit (Ambion) was used to remove contaminating genomic DNA, and a cDNA synthesis kit (Invitrogen, Carlsbad, CA) was used for reverse transcription. Real-time PCR was performed on cDNA using SYBR Green (Applied Biosystems, Foster City, CA). Primers were purchased from Integrated DNA Technologies (Coralville, Iowa). A list of all primer sequences used in this publication is included in Table 1. Mouse primer sequences for the 16 genes used in tumor classification were derived from Cairo et $\mathrm{al}^{7}$ and independently validated in our laboratory. Gapdh, $18 \mathrm{~S} r R N A$, 
and Rhot 2 were used as reference genes for normalization. Gene expression values were obtained by applying the $\Delta \mathrm{Ct}$ method. Expression values for each gene were scaled across samples to establish a mean of 0 and an SD of 1 to allow for direct comparison across samples and genes, as reported by Cairo et al. ${ }^{7}$ These data sets are included in Supplemental Table $\mathrm{S} 1$. The scaled gene expression values from the mouse tumor samples were then combined with the values from the 102 human tumor samples published by Cairo et al. ${ }^{7}$ Unsupervised hierarchical clustering was used to classify the mouse and human tumors. The point-biserial correlation between the proximity (distance) and incidence matrices of the clustering results was calculated to evaluate the fit of the clustering model. The analysis was completed using base $\mathrm{R}$ functions and the add-on packages ComplexHeatmap and $\mathrm{ltm}^{21}$

\section{Statistical Analysis}

Comparisons between treated and control groups were performed by $t$-test using Prism 6 software for Windows version 6 (GraphPad Software, Inc., La Jolla, CA). $P<0.05$ was considered significant.

\section{Results}

Co-Expression of $\beta$-Catenin Point Mutants and Mutant YAP1 Leads to HB Development in Mice

$\Delta$ N90- $\beta$-catenin co-expression with YAP1 in the liver leads to $\mathrm{HB}$ in mice, with complete mortality by 9 to 11 weeks, whereas expression of $\Delta \mathrm{N} 90-\beta$-catenin alone or YAP1 alone does not result in tumor development long-term. ${ }^{14}$ Injection of point mutants S33Y- $\beta$-catenin or S45Y- $\beta$-catenin alone does not yield any liver tumors. ${ }^{22,23}$ Herein, it was studied whether the point mutants of $\beta$-catenin could cooperate with YAP1 and lead to HB just like the deletion mutant. This is relevant because point mutations in CTNNB1 are also frequently observed in HB patients. ${ }^{7}$ S45Y- $\beta$-catenin-S127A-YAP1 $(n=4)$ or S33Y- $\beta$-catenin-S127A-YAP1 $(n=4)$ was expressed using the SB-HTVI in 6-week-old FVB male mice (Figure 1A). Co-expression of YAP1 and point-mutant $\beta$-catenin led to high morbidity and mortality, attributed to enlarged abdominal girth and excessive liver tumor burden in both groups at 8 to 11 weeks after injection, whereas agematched WT controls showed a normal liver weight/body weight ratio at all time points up to 20 weeks of analysis (Figure 1B). Macroscopically, livers from both groups appeared normal, like a WT liver, until 5 weeks after injection. However, at 10 weeks in the S33Y- $\beta$-cateninS127A-YAP1 group and at 9 weeks in the S45Y- $\beta$-cateninS127A-YAP1 group, multiple macroscopic nodules occupying most of the liver surface were evident (Figure 1C). Microscopically, the well-developed tumors observed at these time points were composed of hepatoblasts, which are smaller than hepatocytes and have small round nuclei, with histology of fetal-like or crowded fetal-like HB (Figure 1D).

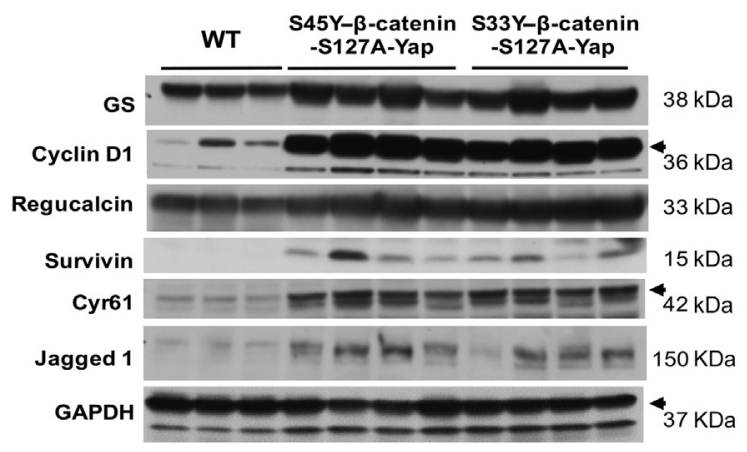

Figure 3 Characterization of hepatoblastoma in S45Y- $\beta$-cateninS127A-YAP1 and S33Y- $\beta$-catenin-S127A-YAP1 mice by analysis of liver lysates. Western blot analysis using lysates from the same groups of mice shows increased levels of $\beta$-catenin targets glutamine synthetase (GS), cyclin D1, and regucalcin, as well as YAP1 targets survivin, Cyr61, and jagged 1, compared with wild-type (WT) livers from age-matched mice. Glyceraldehyde-3-phosphate dehydrogenase (GAPDH) verified comparable loading. Molecular weights are indicated to the right of each blot. Arrows indicate the correct molecular weight band.

Although the tumors lacked severe nuclear pleomorphism, mitotic figures were observed.

To demonstrate that the observed tumors were derived from cells expressing the transgenes, IHC and Western blot analyses were performed for Myc-tag, which is representative of exogenous $\beta$-catenin. Tumors in both $S 45 Y-\beta$-catenin-S127A-YAP1 and S33Y- $\beta$-catenin-S127A-YAP1 groups were strongly positive for Myc-tag by IHC and show strong nuclear staining for YAP1, demonstrating YAP1 activation (Figure 1E). Similarly, whole cell lysates from tumor-bearing livers from both groups showed abundant Myc-tag expression, which was absent in WT livers (Figure 1F). Thus, the combination of YAP1 with either of the point mutants of $\beta$-catenin led to $\mathrm{HB}$ development in mice within a similar time frame as $\Delta \mathrm{N} 90-\beta$-cateninS127A-YAP1 HB tumors.

\section{Characterization of HB in Point-Mutant $\beta$-Catenin- YAP1 Mice}

To further characterize the observed HB, IHC and Western blot analyses were performed for various downstream targets of Wnt and YAP1 signaling pathways. The tumors were strongly positive for the $\beta$-catenin target cyclin D1 compared with normal liver (Figure 2). Likewise, YAP1 targets, including Cyr61, survivin, and jagged 1, were significantly increased, and regucalcin was modestly increased, with expression of activated $\beta$-catenin/YAP1 (Figure 3). As expected, activation of $\beta$-catenin and YAP1 targets, such as cyclin D1, which regulates cell cycle progression, resulted in marked proliferation in $\mathrm{HB}$ from $\beta$-catenin-YAP1 mice, as indicated by Ki-67 positive staining (Figure 2). Thus, co-expression of YAP1 with either point mutant of $\beta$-catenin led to $\mathrm{HB}$, which displayed increased expression of both YAP1 and $\beta$-catenin targets. 
A

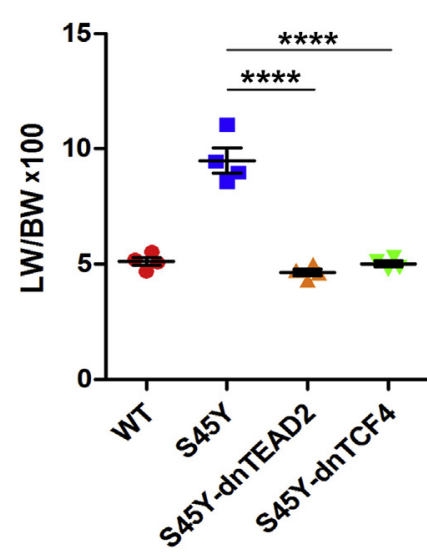

C

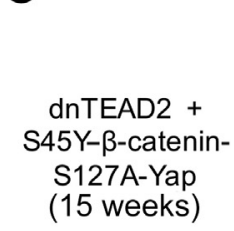

dnTEAD2 +

S33Y- $\beta$-catenin-

S127A-Yap

(15 weeks)

D

dnTCF $4+$

S45Y- $\beta$-catenin-

S127A-Yap

(18 weeks)

dnTCF4 +

S33Y- $\beta$-catenin-

S127A-Yap

(18 weeks)

Gross

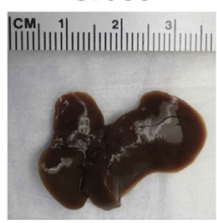

B
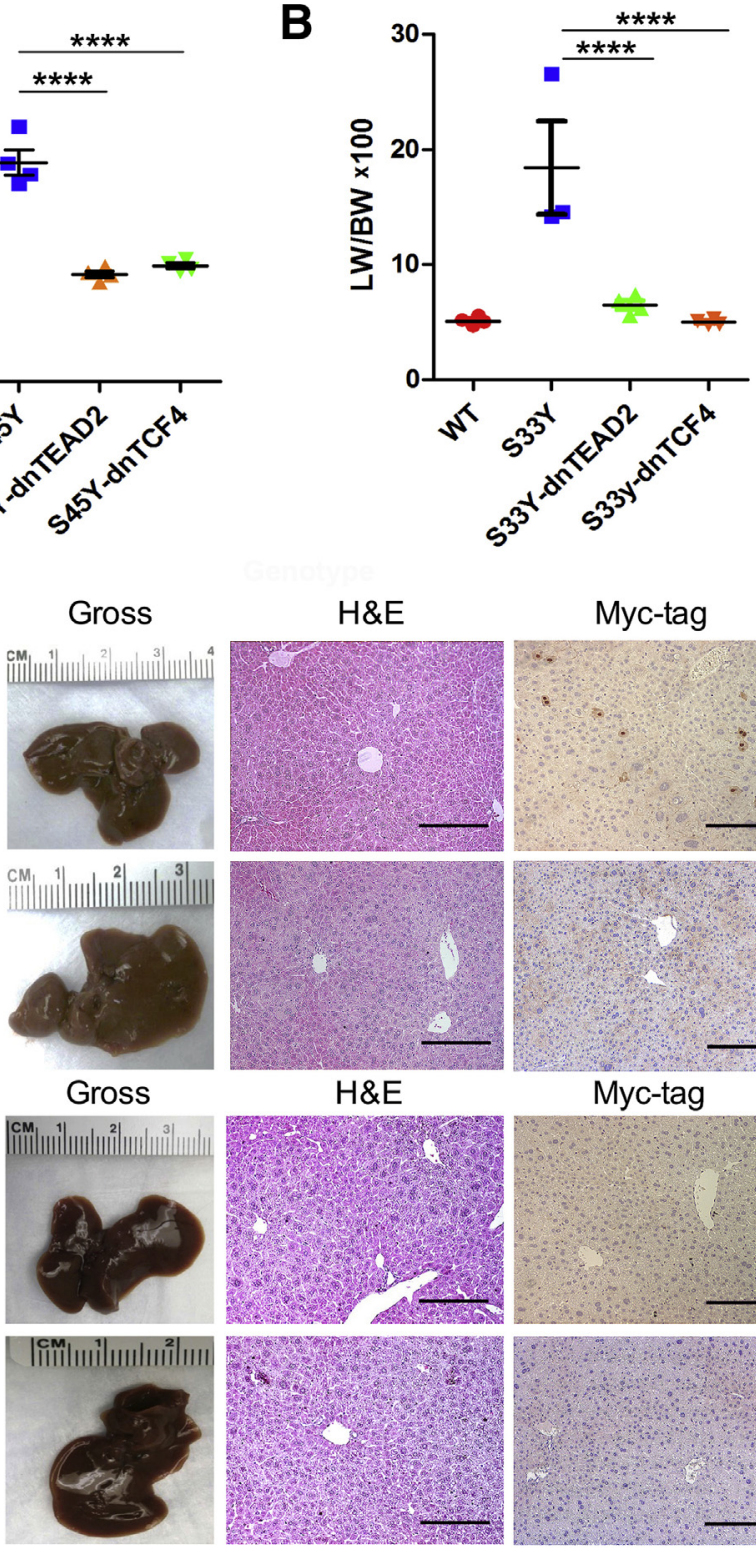

$H \& E$

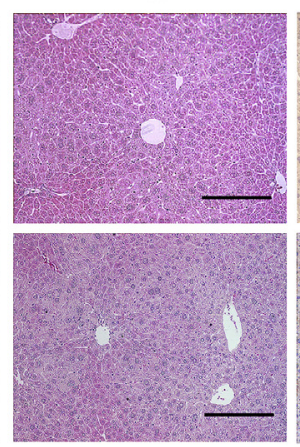

$H \& E$

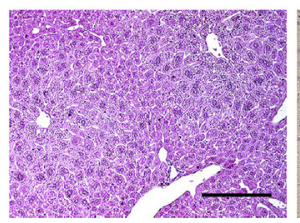

Myc-tag

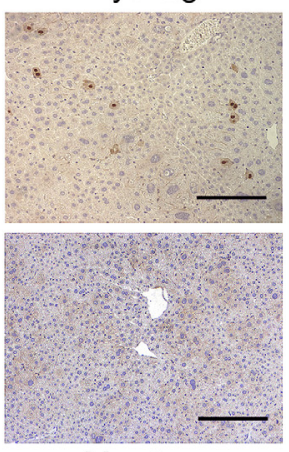

Myc-tag
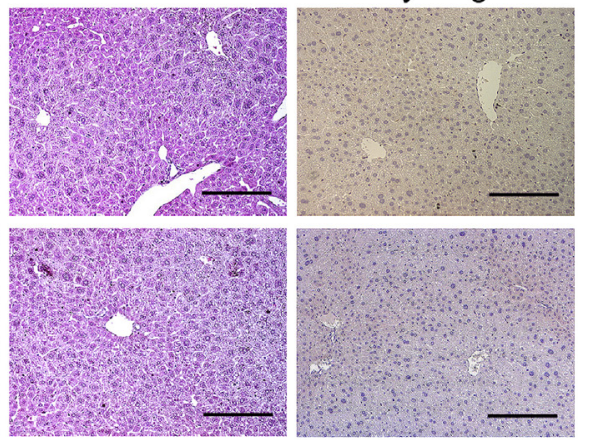

Figure 4 Analysis of hepatoblastoma tumorigenesis in S45Y- $\beta$-catenin-S127A-YAP1 and S33Y- $\beta$-catenin-S127AYAP1 mice in the presence of dnTEAD2 and dnTCF4. A: Liver weight (LW)/body weight (BW) ratio shows approximately $5 \%$ of BW to be contributed by LW in 10- to 12-week-old wildtype (WT) mice, whereas age-matched $S 45 Y-\beta$-cateninS127A-YAP1 co-injected at the time of injection with PT3 plasmid (backbone for dnTCF/dnTEAD) at 8 to 12 weeks after sleeping-beauty hydrodynamic tail vein injection (SB-HTVI) shows, on average, approximately $9 \%$ of BW. Concomitant introduction at the time of SB-HTVI of dnTEAD2 or dnTCF4 led to a highly significant decrease in $\mathrm{LW} / \mathrm{BW}$ ratio comparable to WT, even up to 20 weeks. B: LW/BW ratio shows approximately $5 \%$ of BW to be contributed by LW in 10- to 12-week-old WT mice, whereas age-matched S33Y $-\beta$-catenin-S127A-YAP1 coinjected at the time of injection with PT3 plasmid (backbone for dnTCF/dnTEAD) at 8 to 13 weeks after SB-HTVI shows, on average, approximately $18 \%$ of BW. Concomitant introduction at the time of SB-HTVI of dnTEAD2 or dnTCF4 leads to a highly significant decrease in LW/BW comparable to WT, even up to 20 weeks. C: Gross liver images and hematoxylin and eosin (H\&E) - and Myc-tag-stained sections from dnTEAD2 expressing $S 45 Y-\beta$-catenin-S127A-YAP1 and S33Y- $\beta$-catenin-S127A-YAP1 mice 15 weeks after injection. D: Gross liver images and H\&E- and Myc-tag-stained sections from dnTCF4 expressing $S 45 Y-\beta$-catenin-S127A-YAP1 and S33Y- $\beta$-catenin-S127A-YAP1 mice 18 weeks after injection. $* * * * P<0.0001$. Scale bars $=100 \mu \mathrm{m}(\mathbf{C}$ and $\mathbf{D})$.
Intriguingly, the tumors were positive for GS, a surrogate $\beta$-catenin target (Figure 2). This was unlike the HB occurring in the $\Delta$ N90- $\beta$-catenin-YAP1 model, which expressed GS transiently at the time of tumor initiation, but rapidly evolved into GS-negative tumors. ${ }^{14}$ In the current model with pointmutant $\beta$-catenin, the histology and GS positivity appeared consistent with the characteristics of fetal HB in humans. ${ }^{24}$

\section{Expression of dnTCF4 or dnTEAD2 at the Time of S45Y/S33Y- $\beta$-Catenin-S127A-YAP1 HTVI Prevents Development of HB}

$\beta$-Catenin and YAP1 mainly function as transcriptional coactivators by interacting with the TCF family and TEAD family of DNA-binding transcription factors, respectively, to promote gene expression. ${ }^{25,26}$ To determine whether point- mutant $\beta$-catenin-YAP1 induced HB tumor formation requires interaction with these transcription factors; S45Y$\beta$-catenin-S127A-YAP1 or S33Y- $\beta$-catenin-S127A-YAP1 was codelivered along with dnTCF4 or dnTEAD2 to mouse livers by hydrodynamic tail vein injection using SB-HTVI, as described in Materials and Methods. Both dnTCF4 and dnTEAD 2 bind to $\beta$-catenin and YAP1, respectively, but lack the ability to bind to target gene promoters, thereby blocking their downstream transcriptional activation functions. Co-expression of dnTCF4 or dnTEAD2 along with S45Y- $\beta$ catenin-S127A-YAP1 or S33Y- $\beta$-catenin-S127A-YAP1 completely prevented HB tumorigenesis in mice, even up to 18 weeks after injection, by which time $100 \%$ mortality was evident in the S45Y- $\beta$-catenin-S127A-YAP1 and S33Y- $\beta$ catenin-S127A-YAP1 groups (Figure 4, A and B). This was visible grossly and also histologically, with no microscopic 
A

dnTEAD2 +

S45Y- $\beta$-cateninS127A-Yap

(15 weeks)

dnTEAD2 +

S33Y- $\beta$-catenin-

S127A-Yap

(15 weeks)
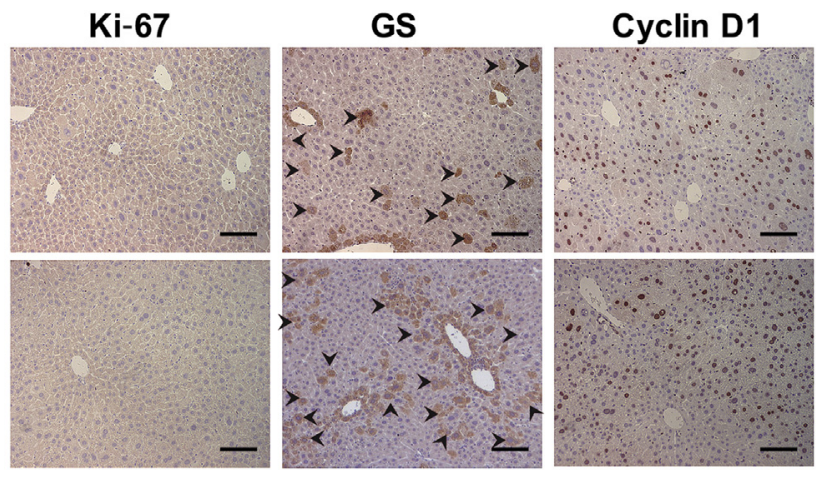

B
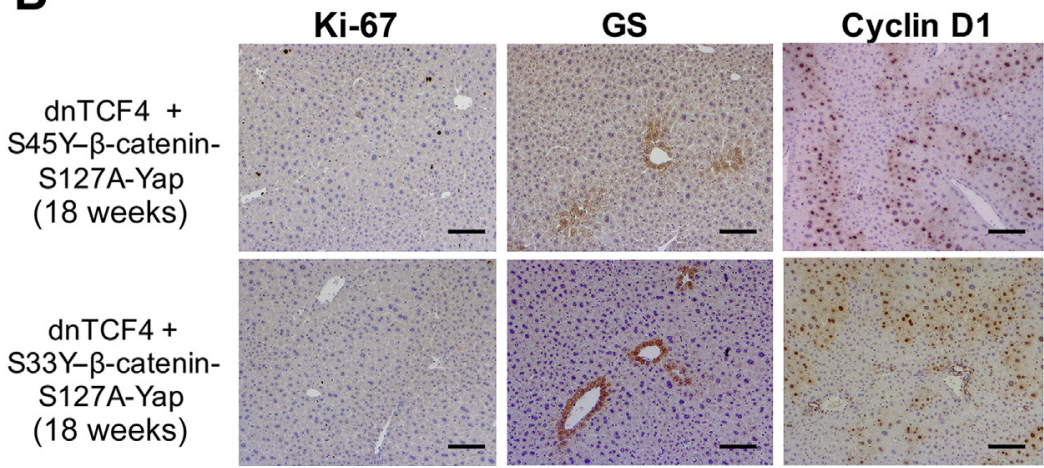

S33Y- $\beta$-catenin-

S127A-Yap

(18 weeks)

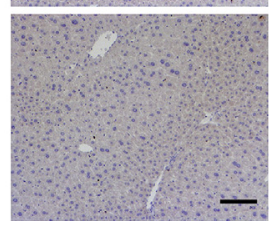

Figure 5 Characterization of livers in dnTEAD2 or dnTCF4 + S45Y- $\beta$-catenin-S127A-YAP1 or S33Y- $\beta$-catenin-S127A-YAP1 mice. A: Representative immunohistochemistry of liver sections shows lack of Ki-67-positive cells in the S45Y- $\beta$-catenin-YAP1-dnTEAD2 and S33Y- $\beta$ catenin-YAP1-dnTEAD2 groups. Cyclin D1 was normalized to its midzonal expression in $\mathrm{S} 45 \mathrm{Y} / \mathrm{S} 33 \mathrm{Y}-\beta$-catenin-YAP1dnTEAD2 groups. Glutamine synthetase (GS) was normalized to its predominantly pericentral expression, with only a few random GS-positive hepatocytes (arrowheads) staying in the S45Y/S33Y- $\beta$-catenin-YAP1-dnTEAD2 groups. More frequent GS-positive cells and occasionally a few cell clusters of GS-positive hepatocytes are seen in the S33Y- $\beta$-catenin-YAP1-dnTEAD2 group. B: Representative immunohistochemistry of liver sections shows lack of Ki$67-$ positive cells in the S45Y- $\beta$-catenin-YAP1-dnTCF4 and S33Y- $\beta$-catenin-YAP1-dnTCF4 groups. Cyclin D1 was normalized to its midzonal expression in the $\mathrm{S} 45 \mathrm{Y} / \mathrm{S} 33 \mathrm{Y}-$ $\beta$-catenin-YAP1-dnTCF4 groups, as was GS to its predominantly pericentral expression. Scale bars $=100 \mu \mathrm{m}(\mathbf{A}$

and $\mathbf{B})$. Original magnification, $\times 100(\mathbf{A}$ and $\mathbf{B})$. foci evident in either of the groups, as seen in representative hematoxylin and eosin staining, as well as the absence of $\mathrm{Ki}$ 67-positive proliferating cells in both models (Figure 4, C and D, and Figure 5). Lack of tumorigenesis was also confirmed by absence of Myc-tag staining by IHC. Only a few isolated Myc-tag-positive cells were seen in the dnTEAD2 groups, and no Myc-tag-positive cells were seen in the dnTCF4 groups (Figure 4, C and D). The addition of the dnTEAD2 and dnTCF4 constructs did not alter the levels of hepatocyte transfection with either $\beta$-catenin or YAP1, showing that the lack of HB tumor growth is not due to a decrease in transfected cells but rather to interference with TEAD2 and TCF4 activity (Supplemental Figure S1). Thus, point-mutant $\beta$-catenin and YAP1 require both of their respective transcription factor partners to stimulate target gene expression and induce HB tumors.

Molecular changes specifically abrogated by lack of $\beta$-catenin-TCF4 or YAP1-TEAD2 interactions were next examined. A notable decrease in cyclin D1, GS, Cyr61, survivin, and jagged 1 was evident in S45Y- $\beta$-catenin-S127AYAP1 cohorts treated with either dnTCF4 or dnTEAD2 compared with advanced tumors (Figures 5 and 6). Similar decreases were observed in these targets in S33Y- $\beta$-cateninS127A-YAP1-dnTCF4. Intriguingly, somewhat divergent molecular alterations were seen in S33Y- $\beta$-catenin-S127AYAP1-dnTEAD2 livers. Sustained GS expression and only a moderate decrease in cyclin D1 (which are both $\beta$-catenin-TCF4 targets) were observed, whereas YAP1 target proteins were dramatically reduced, as in dnTCF4 cohorts
(Figure 5 and Figure 6). These results suggest there may be subtle differences in the gene regulation in each HB model, and further studies are required to determine whether and how $\beta$-catenin and YAP1 cooperate to regulate gene expression. However, in general, these results are consistent with the failure of HB tumors to develop, as the levels of selected $\beta$ catenin and YAP targets are comparable to those in WT mice.

GS Is Expressed Differently in $\triangle N 90-\beta$-Catenin-YAP1 $H B$ versus $S 45 Y / S 33 Y-\beta$-Catenin-S127A-YAP1 HB, although Its Promoter Is Comparably Induced by Deletion versus Point Mutants of $\beta$-Catenin in Vitro

GS expression in HB occurring in $\Delta \mathrm{N} 90-\beta$-catenin-YAP1 versus $\mathrm{S} 45 \mathrm{Y} / \mathrm{S} 33 \mathrm{Y}-\beta$-catenin-S127A-YAP1 was directly compared. Historical samples from $\Delta \mathrm{N} 90-\beta$-cateninYAP1 ${ }^{14}$ and representative samples from S45Y/S33Y $-\beta$ catenin-S127A-YAP1 were stained for GS. The tumors in S45Y/S33Y- $\beta$-catenin-S127A-YAP1 were strongly and uniformly positive for GS versus $\mathrm{HB}$ occurring in $\triangle \mathrm{N} 90-\beta$ catenin-YAP1, which were GS negative (Figure 7A).

It was next investigated whether the point mutant and deletion mutants of $\beta$-catenin used in the HB model exhibited differences in their ability to activate GS expression in primary hepatocytes in vitro. A reporter gene containing the glul $5^{\prime}$ enhancer region, located between -2516 and -2146 upstream from the transcriptional start point (HEV) controlling a firefly luciferase, was transfected into mouse hepatocytes 

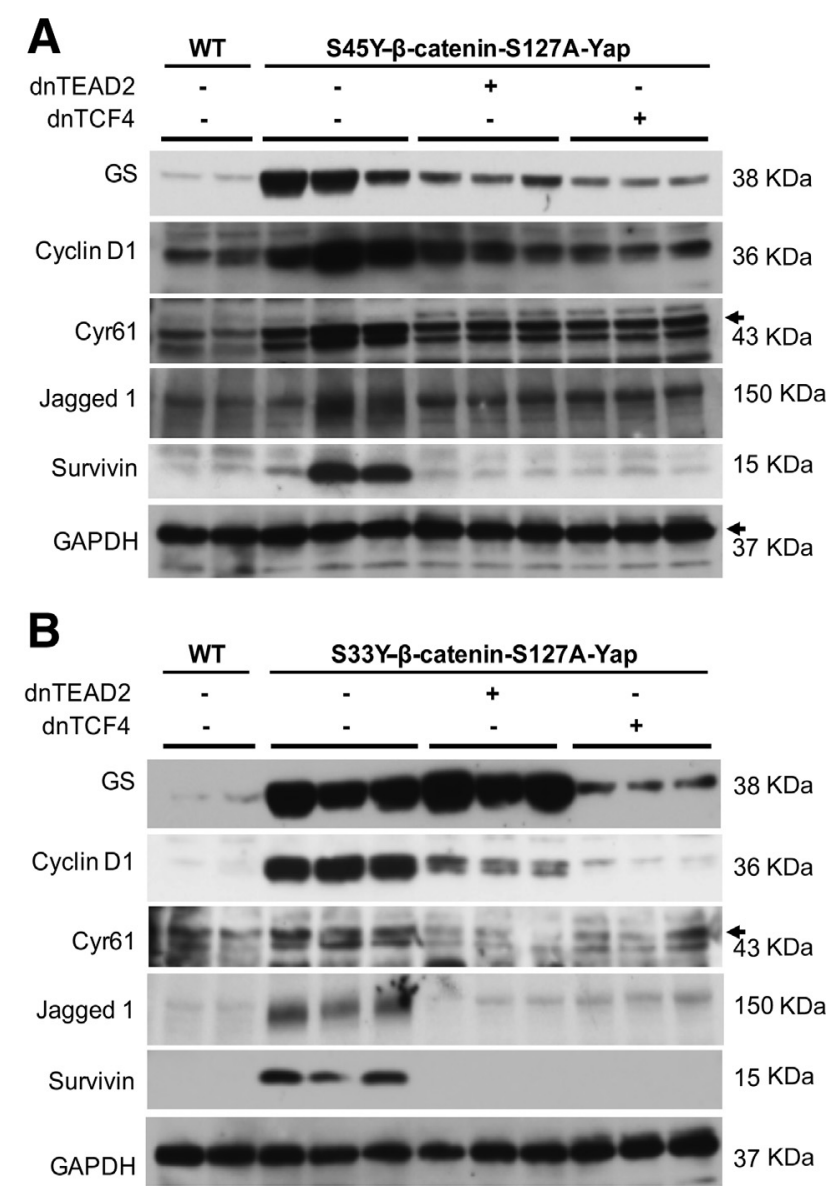

Figure 6 Protein expression of Wnt and YAP1 targets in dnTEAD2 or dnTCF4 + S45Y- $\beta$-catenin-S127A-YAP1 or S33Y- $\beta$-catenin-S127A-YAP1 livers. A: Western blot analysis of $\beta$-catenin targets glutamine synthetase (GS), cyclin D1, and regucalcin, as well as YAP1 targets survivin, Cyr61, and jagged 1, with or without dnTEAD2 or dnTCF4 in S45Y- $\beta$-catenin-S127AYAP1 mice. All targets that were increased in S45Y- $\beta$-catenin-S127A-YAP1 livers relative to wild type (WT) are notably decreased with co-introduction of dnTCF4 or dnTEAD2. Arrows indicate the correct molecular weight. Molecular weights of each protein are indicated to the right. Glyceraldehyde-3-phosphate dehydrogenase (GAPDH) verifies comparable protein loading. B: Western blot analysis of $\beta$-catenin targets GS, cyclin D1, and regucalcin, as well as YAP1 targets survivin, Cyr61, and jagged 1, with or without dnTEAD2 or dnTCF4 in S33Y- $\beta$-catenin-S127A-YAP1 mice. All targets that are increased in S33Y- $\beta$-catenin-S127A-YAP1 livers relative to WT are notably decreased with co-introduction of dnTCF4. However, GS is completely unaffected and cyclin D1 is only modestly affected in S33Y- $\beta$ catenin-S127A-YAP1-dnTEAD2 livers. Arrow indicates the correct molecular weight. Molecular weights of each protein are indicated to the right. GAPDH verifies comparable protein loading.

from two independent liver preparations either without a cotransfected expression plasmid (control) or together with an expression plasmid for $\Delta \mathrm{N} 90-\beta$-catenin or $\mathrm{S} 33 \mathrm{Y}-\beta$ catenin (Figure 7B). Both expression plasmids induced luciferase expression significantly compared with control (Figure 7B). Next, reporter constructs that were missing binding regions for either TCF (HEV-del TCF), STAT5 (HEV-del STAT5), or SP1 (HEV-del SP1) were tested (Figure $7 \mathrm{~B}$ ). In all cases, both $\Delta \mathrm{N} 90-\beta$-catenin and S33Y- $\beta$ catenin caused increases in luciferase expression relative to control, suggesting that their effects are independent of these three transcription factors (Figure 7B). In addition, a reporter gene with the rat glul upstream region from -3780 down to 105 , including the nontranslated mRNA sequence from the glul gene $(H H N)$, was transfected, like above (Figure 7B). In all cases, there was no significant difference in luciferase expression between $\Delta \mathrm{N} 90-\beta$-catenin and $\mathrm{S} 33 \mathrm{Y}-\beta$-catenin constructs (Figure 7B).

\section{Distinct Molecular Classes of HB Are Evident in $\Delta$ N90- $\beta$-Catenin-YAP1 versus S45Y/S33Y- $\beta$-Catenin- S127A-YAP1}

Because GS was expressed differentially between the HB occurring in point versus deletion mutants of $\beta$-catenin, any additional molecular and biological differences in the HB occurring in the two groups were assessed. According to the 2014 international consensus classification of HB, GS expression by immunohistochemistry correlates strongly with the histologic subtype of HB tumors. ${ }^{27}$ Tumors with fetal and well-differentiated fetal morphology are often strongly GS positive, whereas embryonal and pleomorphic tumors have variable GS status and may be GS negative. In addition, Cairo et $\mathrm{al}^{7}$ developed a 16-gene signature that classified 102 human HB tumor samples into two groups: cluster 1 (C1) consisted of predominantly fetal, less proliferative tumors and demonstrated high expression of mature pericentral hepatocyte genes, including GS; whereas cluster 2 (C2) consisted of more aggressive tumors with low GS expression, increased proliferation, and overall worse patient prognosis.

On the basis of the correlation of GS expression with these two patient clusters, the 16-gene signature was used to stratify the point-mutant $\beta$-catenin-YAP and $\Delta N 90-\beta$ catenin-YAP tumor models. Quantitative real-time PCR was used to determine the expression of the 16 genes in both point-mutant $\beta$-catenin models as well as the $\Delta$ N90- $\beta$ catenin tumors, and these data were combined with the scaled gene expression values from the 102 human tumor samples analyzed by Cairo et al. ${ }^{7}$ Unsupervised hierarchical clustering was then used to determine whether point-mutant $\beta$-catenin-YAP tumors and $\Delta$ N90- $\beta$-catenin-YAP tumors were more closely related to $\mathrm{C} 1$ or $\mathrm{C} 2$ patient cases (Figure 8A). Even with the inclusion of mouse tumor data, most of the patient tumors stratified into the same clusters $\mathrm{C} 1$ and $\mathrm{C} 2$, identified by Cairo et $\mathrm{al}^{7}{ }^{7}$ as expected. All five $\Delta$ N90- $\beta$-catenin-YAP tumors tested clustered closely with patient group $\mathrm{C} 2$, as expected on the basis of their GSnegative status. In contrast, both $S 45 \mathrm{Y}-\beta$-catenin-YAP and $\mathrm{S} 33 \mathrm{Y}-\beta$-catenin-YAP tumors exhibited a mixed phenotype. Some tumors clustered closely with $\mathrm{C} 1$ patient tumors, whereas others clustered with a mixed group of both $\mathrm{C} 1$ and $\mathrm{C} 2$ patient tumors. The point-biserial correlation coefficient of the clustering results was determined to be 0.43 , which shows a moderate correlation that could reflect heterogeneity in the tumor gene expression data. 
A

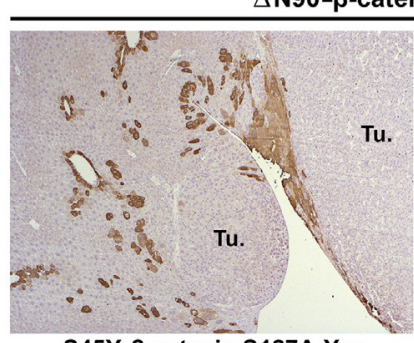

S45Y- $\beta$-catenin-S127A-Yap

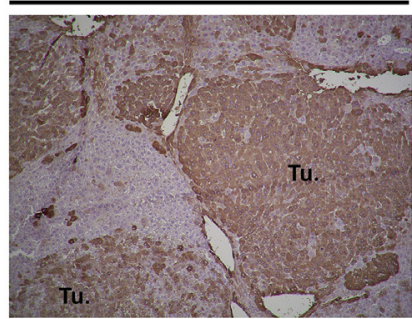

GS

nin-S127A-Yap

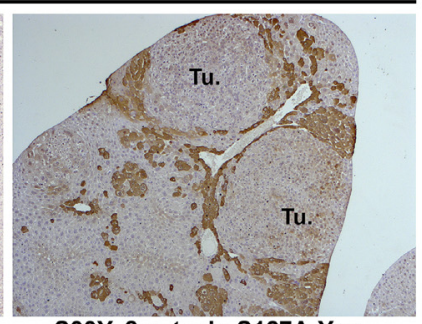

S33Y- $\beta$-catenin-S127A-Yap

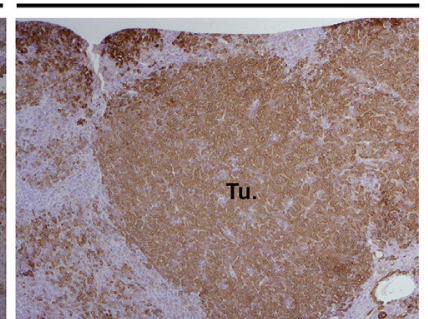

B
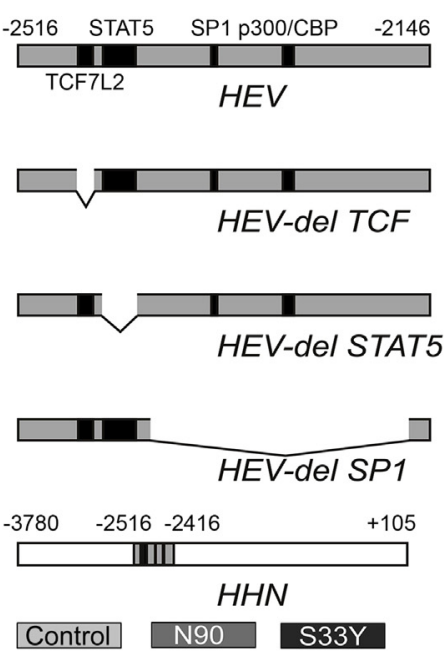
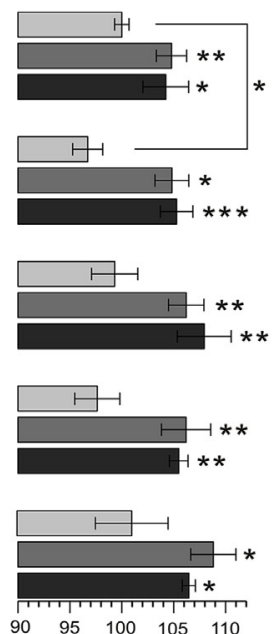

Relative level of expression

compared with HEV (\%)

Figure 7 Glutamine synthetase (GS) expression and regulation in deletion- versus point-mutant hepatoblastoma (HB). A: Two representative images from immunohistochemical staining for GS show HB tumors (Tu.) to be GS negative in the $\Delta$ N90- $\beta$-catenin-YAP1 model (top row). However, Tu. occurring in the S45Y- $\beta$-catenin-YAP1 mice (bottom left panel) or in the S33Y- $\beta$-catenin-YAP1 mice (bottom right panel) are strongly and uniformly GS positive. B: Left panel: Reporter gene assays were performed with $5^{\prime}$ sequences from the rat Glul gene containing the Glul $5^{\prime}$-enhancer region located between -2516 and -2146 upstream from the transcriptional start point (HEV) and reporter genes with deletions within this region [HEV-del TCF, HEV-del STAT5, and HEV-del specificity protein 1 (SP1)] controlling a firefly luciferase. These reporters were transfected into mouse hepatocytes from two independent liver preparations either without a cotransfected expression plasmid (control) or together with a $\Delta \mathrm{N} 90-\beta$-catenin expression plasmid or an S33Y- $\beta$-catenin expression plasmid. In addition, a reporter gene with the rat Glul upstream region from -3780 down to 105, including the non-translated mRNA sequence from the Glul gene $(H H N)$, was transfected. The relative level of expression was determined from a cotransfected reference reporter expressing Renilla luciferase. Right panel: All values depicted are normalized to the relative expression of the HEV reporter transfected without an expression plasmid for $\beta$-catenin (set as $100 \%$ ). Data are from two to three independent transfection experiments performed with each of the two mouse hepatocyte preparations. Data are expressed as means \pm SD. ${ }^{*} P<0.05,{ }^{* *} P<0.01$, and ${ }^{* * *} P<0.001$ versus the corresponding control for each reporter gene or HEV + control versus HEV-del TCF + control ( $t$-test). Original magnification, $\times 50(A)$. CBP, CREB-binding protein.

The expression of GS in these samples was quantitated via quantitative RT-PCR (Figure 8B). As expected, $\Delta$ N90- $\beta$-catenin-YAP tumors showed only a small increase in GS expression compared with WT mice. Although both point-mutant $\beta$-catenin-YAP HB tumors tend to show much higher expression levels of GS than WT, there was some variability in expression that may be due to tissue heterogeneity in the tumor samples. This heterogeneity may contribute to some variability in the clustering analysis.

The expression patterns of the enzyme cytochrome 2E1, which is normally expressed in the pericentral zone of the liver lobule and was consistently highly expressed in $\mathrm{C} 1$ patient tumors, were further examined. $\Delta$ N90- $\beta$-catenin-YAP tumor showed mostly low expression levels of cytochrome 2E1, whereas S45Y- $\beta$-catenin-YAP and S33Y- $\beta$-catenin-YAP tumors showed mixed levels of expression, with areas of significantly elevated cytochrome 2E1 expression (Figure 8C). These results illustrate the significant genetic heterogeneity in the point-mutant $\beta$-catenin/YAP HB tumors that was reflected in the clustering analysis.

These results show that, although different mutations in $\beta$ catenin can all promote HB tumor development, there are important underlying signaling differences in the tumor biology that should be further distinguished and may correlate with tumor behavior and prognosis.

\section{Discussion}

The cellular and molecular pathways involved in $\mathrm{HB}$ tumorigenesis are poorly understood and require further investigation to aid in diagnosis, treatment, and prolongation of overall survival. Multiple studies have demonstrated aberrant $\mathrm{Wnt} / \beta$-catenin signaling as a key player in $\mathrm{HB}$ pathogenesis. $^{5,7,10,28,29} \beta$-Catenin alone is deemed insufficient for HB pathogenesis because expression of a truncated form of $\beta$-catenin, either in a transgenic mouse, delivered by adenovirus, or delivered by SB-HTVI, does not lead to tumor formation. ${ }^{14,30,31}$ However, co-expression of the deletion mutant $\Delta$ N90- $\beta$-catenin with YAP1 led rapidly to HB development. ${ }^{14}$ Our current study shows that, like the deletion mutant of $\beta$-catenin, two point mutants of $\beta$-catenin, S33Y and S45Y, also cooperate effectively with YAP1 to lead to HB. As expected, $\beta$-catenin and YAP1 signaling activity was evident in the S45Y- $\beta$-catenin-S127A-YAP1 and S33Y- $\beta$-catenin-S127A-YAP1 groups, as shown by IHC and Western blot analyses as well as up-regulation of traditional Wnt and YAP1 targets. The relevance of the respective transcription factor partners TCF4 and TEAD2 in mediating $\beta$-catenin and YAP1 activity in HB development has been demonstrated. ${ }^{25,26}$ Suppression of $\beta$-catenin and YAP signaling by either dnTCF4 or dnTEAD2 in the S45Y- $\beta$ - 
A

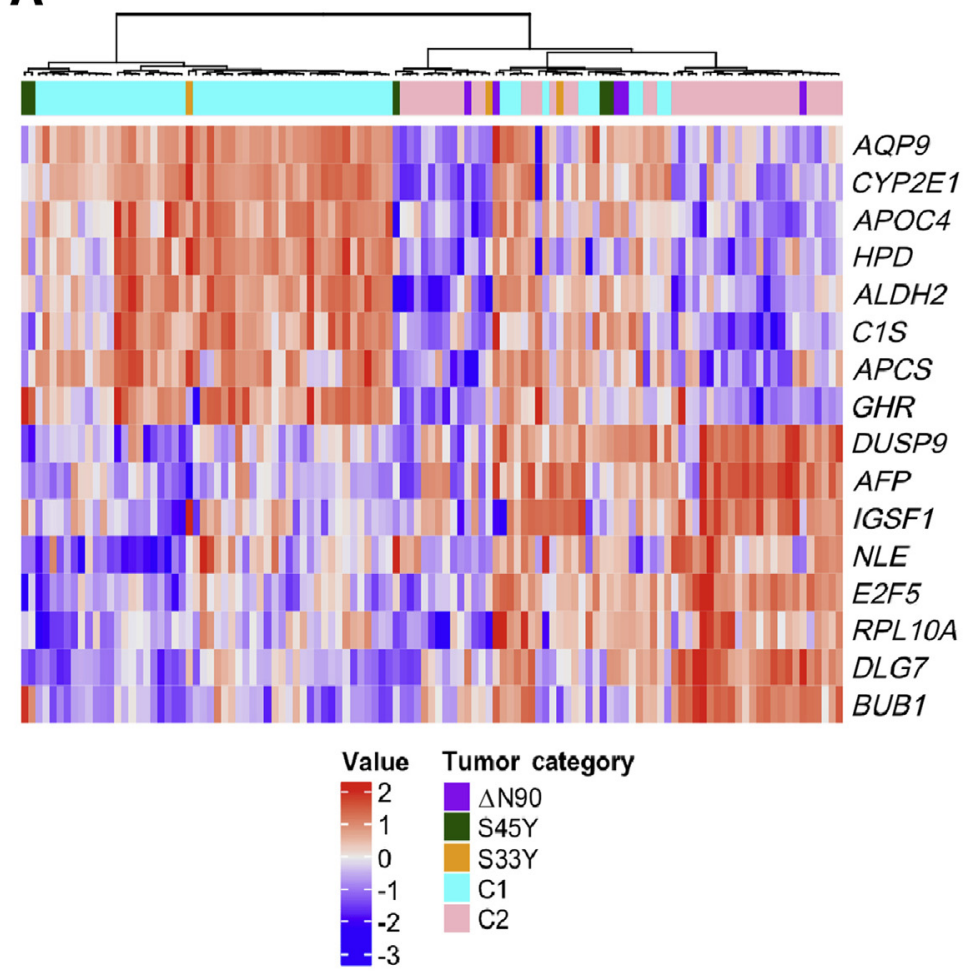

B

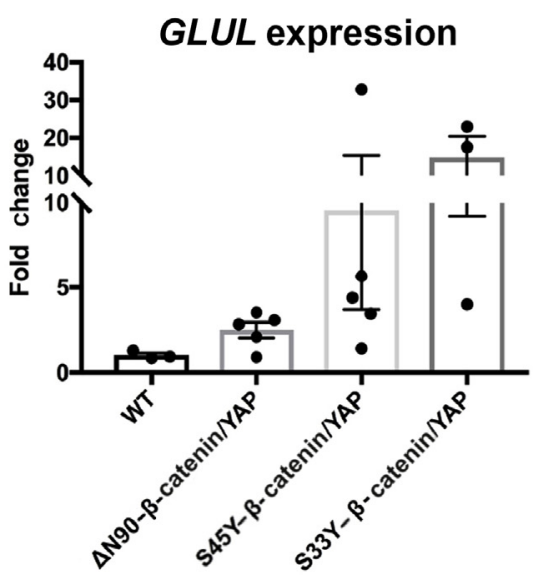

C

N90- $\beta$-catenin-S127A-YAP1

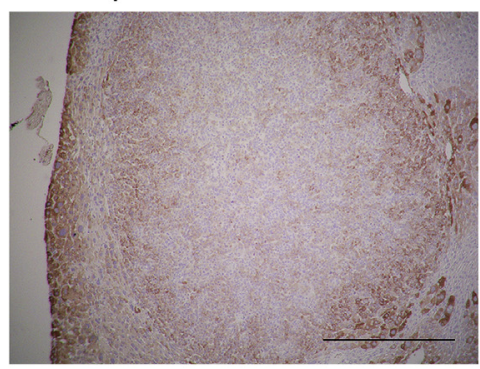

S45Y- $\beta$-catenin-S127A-YAP1

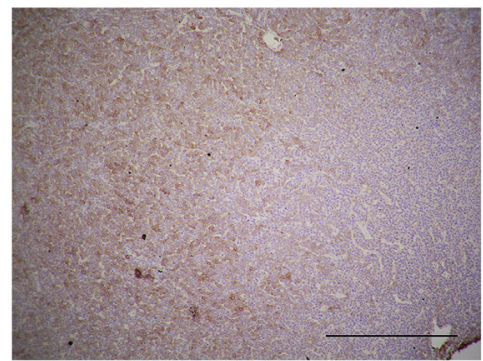

\section{S33Y- $\beta$-catenin-S127A-YAP1}

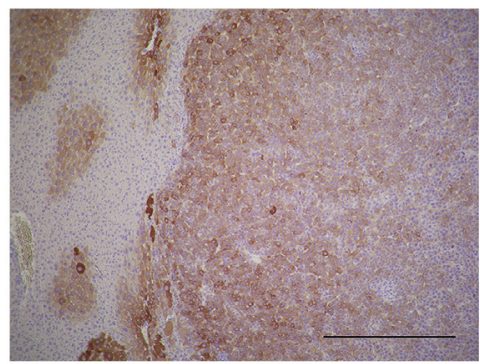

Figure 8 Differential glutamine synthetase (GS) expression and molecular features of deletion- versus point-mutant hepatoblastoma (HB) tumors. A: The heat map shows the scaled gene expression values for the 16 genes labeled at right in each tumor sample, including 102 human HB samples first analyzed by Cairo et al, ${ }^{7}$ and several mouse HB tumors from our laboratory: five $\triangle N 90-\beta$-catenin-YAP1, five S45Y- $\beta$-catenin-YAP1, and three S33Y- $\triangle N 90-\beta$-catenin-YAP1 tumors. All tumors are annotated by tumor category. Human tumors are classified as cluster 1 (C1) and cluster 2 (C2), as determined originally by Cairo et al. ${ }^{7}$ Unsupervised hierarchical clustering results are shown by the dendrogram at the top of the heat map. Most of the C1 human tumors cluster together, as do the C2 human tumors, thus correlating well with the original analysis. B: mRNA expression of Glul, encoding for GS, was determined by quantitative RT-PCR on whole liver lysates from all three HB tumor models and wild-type (WT) FVB mice for comparison. C: Representative images of immunohistochemistry staining for the pericentral enzyme cytochrome $2 \mathrm{E} 1$ in tumors from all three tumor models: $\Delta N 90-\beta$-catenin-YAP1, S45Y- $\beta$-catenin-YAP1, and S33Y- $\Delta N 90-\beta$-cateninYAP1. Scale bars $=500 \mu \mathrm{m}(\mathbf{C})$.

catenin-S127A-YAP1 or S33Y- $\beta$-catenin-S127A-YAP1 HB model completely prevented tumor formation. The dominant-negative forms of TCF4 and TEAD2 may outcompete other members of their respective transcription factor families, and thus the contributions of other members of the TCF or TEAD families in HB pathogenesis in these models cannot be ruled out.

One intriguing observation was the difference in GS expression between the deletion and point-mutant tumor models. GS is a well-known target of $\beta$-catenin, and many liver tumors that demonstrate $\beta$-catenin activation, including hepatocellular carcinoma, adenomas, and $\mathrm{HB}$, are often also GS positive. ${ }^{6}$ GS is used as a biomarker representative of $\beta$ catenin activity as well as of well-differentiated liver tumors. ${ }^{27}$ More important, although most HB tumors of all subtypes demonstrate $\beta$-catenin activation, the more highly differentiated tumors with fetal or crowded fetal histology are often strongly GS positive, whereas embryonal tumors or those with mesenchymal components are often GS negative. ${ }^{7,24,27}$ Still other studies have reported a range of heterogeneous GS staining patterns across HB subtypes. ${ }^{10,32}$ The results of this study are generally in concordance with 
these observations, as the GS-negative deletion mutant $\mathrm{HB}$ model exhibited molecular similarities with the GS-negative, less-differentiated patient cohort previously described, ${ }^{7}$ although the GS-positive HB models showed a mixed phenotype. These results, along with the previous literature, ${ }^{7,10,24,27,32}$ show that GS is not a surrogate biomarker for all forms of $\beta$-catenin activation, and the type of $\beta$-catenin mutation may play an important role in the molecular pathogenesis of each tumor. Indeed, different target genes of the Wnt pathway have been identified in different subsets of $\mathrm{HB}$ because of deletions versus point mutations. ${ }^{7,10,24,28,33,34}$ Studies have also shown GS to be more closely correlated with $\beta$-catenin activation because of $C T N N B 1$ mutations as opposed to other mechanisms, such as mutations in upstream regulators of the Wnt pathway. ${ }^{35,36}$ Thus, care should be taken when using GS as a biomarker for tumor diagnosis and classification in $\mathrm{HB}$, and $\beta$-catenin activation should be assessed separately.

Large deletion mutations encompassing exon 3 have been more often associated with fetal HB. ${ }^{7,10}$ Similarly, small deletions or point mutations are associated more with embryonal or other subtypes of HB. In our HB mouse models driven by either deletion or point-mutant $\beta$-catenin in combination with YAP1, the tumors demonstrate mostly fetal histology with some heterogeneity, with areas of crowded fetal and embryonal histology appearing in all three models. ${ }^{10}$

Previous studies have found other significant differences in liver tumor or cell line characteristics associated with different types of $\beta$-catenin mutations. For example, a study of four HB cell lines demonstrated differences in metabolic activity that correlated with the presence of exon 3 deletion mutants versus point mutants of $\beta$-catenin. ${ }^{33}$ Another study has shown that both large deletions and S33 point mutants exhibit strong $\beta$-catenin activation and induction of TopFlash (Addgene, Cambridge, MA) activity and GS expression in vitro, whereas $\mathrm{S} 45$ point mutants exhibit only weak $\beta$-catenin activation and low induction of GS expression compared with WT. ${ }^{9}$ At the same time, the authors also found that gene duplication of mutations affecting S45 could result in overall comparable $\beta$-catenin activity as large deletions and S33 point mutants. No major difference was observed in the timeline of tumor development, overall tumor burden, or any other notable differences between the two point mutants, suggesting that both point mutants provide sufficient $\beta$-catenin activity to drive $\mathrm{HB}$ tumorigenesis in combination with YAP1.

Furthermore, our in vitro findings are consistent with previous results, ${ }^{9}$ as we show that both the deletion and S33 point mutants of $\beta$-catenin exhibit comparable induction of luciferase expression, driven by the $5^{\prime}$ upstream regulatory region of the glul gene. Interestingly, this up-regulation did not depend on known DNA-binding regions that interact with TCF4 (TCF7L2), suggesting that $\beta$-catenin may regulate GS expression through an alternative mechanism that remains to be determined.
Do point and deletion mutant of $\beta$-catenin have different molecular targets that could contribute to differences in tumor biology? We recently compared the abilities of $S 45 \mathrm{Y}-\beta$-catenin and $\Delta \mathrm{N} 90-\beta$-catenin to cooperate with the c-Met proto-oncogene in inducing hepatocellular carcinoma. ${ }^{37}$ We showed their comparable oncogenic potential, demonstrable by a similar timeline of induced tumors and similarly altered target genes, albeit with differences in extent of changes, suggesting that both forms of oncogenic $\beta$-catenin at least interact comparably with c-Met. Clearly, in the current HB model, there appear to be subtle differences in how deletion- versus point-mutant $\beta$-catenin may be interacting with YAP1 to differentially regulate some genes, like glul, although the specific mechanism remains unclear. Overall, though, both forms of $\beta$-catenin are comparably competent to interact with YAP1 to induce HB; and future studies will be essential to specifically identify differentially regulated genes that may contribute to differences in HB histology.

To interpret these results in the context of $\mathrm{HB}$, the impact of potential interactions between $\beta$-catenin and YAP1 in regulating gene expression in $\mathrm{HB}$ must be considered. YAP1 has been shown to regulate GS expression in the zebrafish liver, although this has not been shown in the mouse. ${ }^{38}$ In S33Y- $\beta$-catenin-S127A-YAP1 tumors, treatment with dnTEAD2 did not decrease GS expression, despite the lack of tumor formation, whereas dnTCF4 successfully blocked GS expression, suggesting that $\beta$-catenin independently drives GS expression in this case. However, in S45Y- $\beta$-catenin-S127A-YAP1 tumors, treatment with dnTEAD2 produced a strong decrease in GS expression comparable to that achieved by dnTCF4, suggesting that either YAP1 or both YAP1/ $\beta$-catenin may contribute to GS expression in this model. Further studies are needed to determine the specific nature of the interactions between $\beta$-catenin and YAP1 in HB and how different mutations of $\beta$-catenin may alter this dynamic.

Overall, our study shows that, although several different types of $\beta$-catenin mutations can successfully drive $\mathrm{HB}$ tumorigenesis in combination with YAP1 in a mouse model, there may be subtle differences in $\beta$-catenin signaling associated with different mutation types that impact tumor behavior and require further investigation in the context of HB.

\section{Supplemental Data}

Supplemental material for this article can be found at https://doi.org/10.1016/j.ajpath.2019.02.002.

\section{References}

1. Czauderna P, Haeberle B, Hiyama E, Rangaswami A, Krailo M, Maibach R, Rinaldi E, Feng Y, Aronson D, Malogolowkin M, Yoshimura K, Leuschner I, Lopez-Terrada D, Hishiki T, Perilongo G, von Schweinitz D, Schmid I, Watanabe K, Derosa M, Meyers R: The Children's Hepatic tumors International Collaboration (CHIC): novel 
global rare tumor database yields new prognostic factors in hepatoblastoma and becomes a research model. Eur J Cancer 2016, 52:92-101

2. Pappo AS, Furman WL, Schultz KA, Ferrari A, Helman L, Krailo MD: Rare tumors in children: progress through collaboration. J Clin Oncol 2015, 33:3047-3054

3. Perilongo G, Malogolowkin M, Feusner J: Hepatoblastoma clinical research: lessons learned and future challenges. Pediatr Blood Cancer 2012, 59:818-821

4. Czauderna P, Lopez-Terrada D, Hiyama E, Haberle B, Malogolowkin MH, Meyers RL: Hepatoblastoma state of the art: pathology, genetics, risk stratification, and chemotherapy. Curr Opin Pediatr 2014, 26:19-28

5. Koch A, Denkhaus D, Albrecht S, Leuschner I, von Schweinitz D, Pietsch T: Childhood hepatoblastomas frequently carry a mutated degradation targeting box of the beta-catenin gene. Cancer Res 1999, 59:269-273

6. Monga SP: Beta-Catenin signaling and roles in liver homeostasis, injury, and tumorigenesis. Gastroenterology 2015, 148:1294-1310

7. Cairo S, Armengol C, De Reynies A, Wei Y, Thomas E, Renard CA, et al: Hepatic stem-like phenotype and interplay of Wnt/beta-catenin and Myc signaling in aggressive childhood liver cancer. Cancer Cell 2008, 14:471-484

8. Okabe H, Kinoshita H, Imai K, Nakagawa S, Higashi T, Arima K, Uchiyama H, Ikegami T, Harimoto N, Itoh S, Ishiko T, Yoshizumi T, Beppu T, Monga SP, Baba H, Maehara Y: Diverse basis of betacatenin activation in human hepatocellular carcinoma: implications in biology and prognosis. PLoS One 2016, 11:e0152695

9. Rebouissou S, Franconi A, Calderaro J, Letouzé E, Imbeaud S, Pilati C, Nault J-C, Couchy G, Laurent A, Balabaud C, BioulacSage P, Zucman-Rossi J: Genotype-phenotype correlation of CTNNB1 mutations reveals different B-catenin activity associated with liver tumor progression. Hepatology 2016, 64:2047-2061

10. Lopez-Terrada D, Gunaratne PH, Adesina AM, Pulliam J, Hoang DM, Nguyen Y, Mistretta TA, Margolin J, Finegold MJ: Histologic subtypes of hepatoblastoma are characterized by differential canonical Wnt and Notch pathway activation in DLK+ precursors. Hum Pathol 2009, 40:783-794

11. Li H, Wolfe A, Septer S, Edwards G, Zhong X, Abdulkarim AB, Ranganathan S, Apte U: Deregulation of Hippo kinase signalling in human hepatic malignancies. Liver Int 2012, 32:38-47

12. Patel SH, Camargo FD, Yimlamai D: Hippo signaling in the liver regulates organ size, cell fate, and carcinogenesis. Gastroenterology 2017, 152:533-545

13. Azzolin L, Panciera T, Soligo S, Enzo E, Bicciato S, Dupont S, Bresolin S, Frasson C, Basso G, Guzzardo V, Fassina A, Cordenonsi M, Piccolo S: YAP/TAZ incorporation in the beta-catenin destruction complex orchestrates the Wnt response. Cell 2014, 158: $157-170$

14. Tao J, Calvisi DF, Ranganathan S, Cigliano A, Zhou L, Singh S, Jiang L, Fan B, Terracciano L, Armeanu-Ebinger S, Ribback S, Dombrowski F, Evert M, Chen X, Monga SPS: Activation of $\beta$-catenin and Yap1 in human hepatoblastoma and induction of hepatocarcinogenesis in mice. Gastroenterology 2014, 147:690-701

15. Molina L, Bell D, Tao J, Preziosi M, Pradhan-Sundd T, Singh S, Poddar M, Luo J, Ranganathan S, Chikina M, Monga SP: Hepatocyte-derived lipocalin 2 is a potential serum biomarker reflecting tumor burden in hepatoblastoma. Am J Pathol 2018, 188: 1895-1909

16. Kanefuji T, Yokoo T, Suda T, Abe H, Kamimura K, Liu D: Hemodynamics of a hydrodynamic injection. Mol Ther Methods Clin Dev 2014, 1:14029

17. Bonamassa B, Hai L, Liu D: Hydrodynamic gene delivery and its applications in pharmaceutical research. Pharm Res 2011, 28:694-701

18. Fahrner J, Labruyere WT, Gaunitz C, Moorman AF, Gebhardt R, Lamers WH: Identification and functional characterization of regulatory elements of the glutamine synthetase gene from rat liver. Eur J Biochem 1993, 213:1067-1073
19. Werth M, Gebhardt R, Gaunitz F: Hepatic expression of glutamine synthetase in rats is controlled by STAT5 and TCF transcription factors. Hepatology 2006, 44:967-975

20. Nordeen SK: Luciferase reporter gene vectors for analysis of promoters and enhancers. Biotechniques 1988, 6:454-458

21. Gu Z, Eils R, Schlesner M: Complex heatmaps reveal patterns and correlations in multidimensional genomic data. Bioinformatics 2016, $32: 2847-2849$

22. Tao J, Xu E, Zhao Y, Singh S, Li X, Couchy G, Chen X, ZucmanRossi J, Chikina M, Monga SP: Modeling a human hepatocellular carcinoma subset in mice through coexpression of met and pointmutant beta-catenin. Hepatology 2016, 64:1587-1605

23. Tao J, Zhang R, Singh S, Poddar M, Xu E, Oertel M, Chen X, Ganesh S, Abrams M, Monga SP: Targeting beta-catenin in hepatocellular cancers induced by coexpression of mutant beta-catenin and KRas in mice. Hepatology 2017, 65:1581-1599

24. Armengol C, Cairo S, Fabre M, Buendia MA: Wnt signaling and hepatocarcinogenesis: the hepatoblastoma model. Int J Biochem Cell Biol 2011, 43:265-270

25. Behrens J, von Kries JP, Kuhl M, Bruhn L, Wedlich D, Grosschedl R, Birchmeier W: Functional interaction of beta-catenin with the transcription factor LEF-1. Nature 1996, 382:638-642

26. Wu S, Liu Y, Zheng Y, Dong J, Pan D: The TEAD/TEF family protein Scalloped mediates transcriptional output of the Hippo growthregulatory pathway. Dev Cell 2008, 14:388-398

27. Lopez-Terrada D, Alaggio R, de Davila MT, Czauderna P, Hiyama E, Katzenstein H, Leuschner I, Malogolowkin M, Meyers R, Ranganathan S, Tanaka Y, Tomlinson G, Fabre M, Zimmermann A, Finegold MJ; Children's Oncology Group Liver Tumor Committee: Towards an international pediatric liver tumor consensus classification: proceedings of the Los Angeles COG liver tumors symposium. Mod Pathol 2014, 27:472-491

28. Adesina AM, Lopez-Terrada D, Wong KK, Gunaratne P, Nguyen Y, Pulliam J, Margolin J, Finegold MJ: Gene expression profiling reveals signatures characterizing histologic subtypes of hepatoblastoma and global deregulation in cell growth and survival pathways. Hum Pathol 2009, 40:843-853

29. Takayasu H, Horie H, Hiyama E, Matsunaga T, Hayashi Y, Watanabe Y, Suita S, Kaneko M, Sasaki F, Hashizume K, Ozaki T, Furuuchi K, Tada M, Ohnuma N, Nakagawara A: Frequent deletions and mutations of the beta-catenin gene are associated with overexpression of cyclin D1 and fibronectin and poorly differentiated histology in childhood hepatoblastoma. Clin Cancer Res 2001, 7: 901-908

30. Cadoret A, Ovejero C, Saadi-Kheddouci S, Souil E, Fabre M, Romagnolo B, Kahn A, Perret C: Hepatomegaly in transgenic mice expressing an oncogenic form of beta-catenin. Cancer Res 2001, 61: $3245-3249$

31. Harada N, Miyoshi H, Murai N, Oshima H, Tamai Y, Oshima M, Taketo MM: Lack of tumorigenesis in the mouse liver after adenovirus-mediated expression of a dominant stable mutant of betacatenin. Cancer Res 2002, 62:1971-1977

32. Schmidt A, Braeuning A, Ruck P, Seitz G, Armeanu-Ebinger S, Fuchs J, Warmann SW, Schwarz M: Differential expression of glutamine synthetase and cytochrome P450 isoforms in human hepatoblastoma. Toxicology 2011, 281:7-14

33. Crippa S, Ancey PB, Vazquez J, Angelino P, Rougemont AL, Guettier C, Zoete V, Delorenzi M, Michielin O, Meylan E: Mutant CTNNB1 and histological heterogeneity define metabolic subtypes of hepatoblastoma. EMBO Mol Med 2017, 9:1589-1604

34. Lade A, Ranganathan S, Luo J, Monga SP: Calpain induces N-terminal truncation of beta-catenin in normal murine liver development: diagnostic implications in hepatoblastomas. J Biol Chem 2012, 287: 22789-22798

35. Cieply B, Zeng G, Proverbs-Singh T, Geller DA, Monga SP: Unique phenotype of hepatocellular cancers with exon-3 mutations in betacatenin gene. Hepatology 2009, 49:821-831 
36. Schulze K, Imbeaud S, Letouze E, Alexandrov LB, Calderaro J, Rebouissou S, Couchy G, Meiller C, Shinde J, Soysouvanh F, Calatayud AL, Pinyol R, Pelletier L, Balabaud C, Laurent A, Blanc JF, Mazzaferro V, Calvo F, Villanueva A, Nault JC, Bioulac-Sage P, Stratton MR, Llovet JM, Zucman-Rossi J: Exome sequencing of hepatocellular carcinomas identifies new mutational signatures and potential therapeutic targets. Nat Genet 2015, 47:505-511

37. Qiao Y, Xu M, Tao J, Che L, Cigliano A, Monga SP, Calvisi DF, Chen X: Oncogenic potential of N-terminal deletion and S45Y mutant beta-catenin in promoting hepatocellular carcinoma development in mice. BMC Cancer 2018, 18:1093

38. Cox AG, Hwang KL, Brown KK, Evason K, Beltz S, Tsomides A, O'Connor K, Galli GG, Yimlamai D, Chhangawala S, Yuan M, Lien EC, Wucherpfennig J, Nissim S, Minami A, Cohen DE, Camargo FD, Asara JM, Houvras Y, Stainier DYR, Goessling W: Yap reprograms glutamine metabolism to increase nucleotide biosynthesis and enable liver growth. Nat Cell Biol 2016, 18: 886-896 